\title{
Correction: Mutation of the BRCA1 SQ-cluster results in aberrant mitosis, reduced homologous recombination, and a compensatory increase in non-homologous end joining
}

\section{Jason M. Beckta, Seth M. Dever, Nisha Gnawali, Ashraf Khalil, Amrita Sule, Sarah E. Golding, Elizabeth Rosenberg, Aarthi Narayanan, Kylene Kehn-Hall, Bo Xu, Lawrence F. Povirk and Kristoffer Valerie}

Present: Due to an error made during manuscript preparation, one of Dr. Ashraf Khalil's affiliations was omitted from the final version of the article.

Corrected: The correct affiliation list for this manuscript is provided below. Publisher sincerely apologizes for this oversight.

Original article: Oncotarget. 2015; 6(29): 27674-87. doi: 10.18632/oncotarget.4876.

\footnotetext{
Jason M. Beckta ${ }^{1,2, *}$, Seth M. Dever ${ }^{1,2, *}$, Nisha Gnawali ${ }^{1}$, Ashraf Khalil ${ }^{1,7}$, Amrita Sule $^{1,2}$, Sarah E. Golding ${ }^{1}$, Elizabeth Rosenberg ${ }^{1}$, Aarthi Narayanan ${ }^{3}$, Kylene KehnHall ${ }^{3}$, Bo Xu ${ }^{4}$, Lawrence F. Povirk ${ }^{5}$, Kristoffer Valerie ${ }^{1,2,6}$

${ }^{1}$ Department of Radiation Oncology, Virginia Commonwealth University, Richmond, VA, USA

2 Department of Biochemistry and Molecular Biology, Virginia Commonwealth University, Richmond, VA, USA

${ }^{3}$ National Center for Biodefense and Infectious Diseases, George Mason University, Manassas, VA, USA

${ }^{4}$ Cancer Research Department, Southern Research Institute, Birmingham, AL, USA

${ }^{5}$ Department of Pharmacology and Toxicology, Virginia Commonwealth University, Richmond, VA, USA

${ }^{6}$ Massey Cancer Center, Virginia Commonwealth University, Richmond, VA, USA

${ }^{7}$ Department of Biochemistry, National Liver Institute, Menoufiya University, Egypt

* These authors have contributed equally to this work
} 